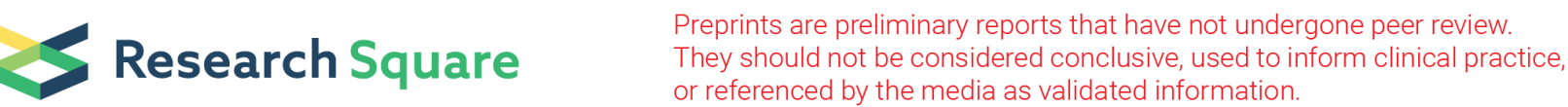

\section{Texture Analysis of Non-Contrast T2-Weighted Cardiovascular Magnetic Resonance Imaging to Discriminate Between Cardiac Amyloidosis and Hypertrophic Cardiomyopathy}

Shan Huang

West China Hospital of Sichuan University

Ke Shi

West China Hospital of Sichuan University

Yi Zhang

West China Hospital of Sichuan University

Wei-feng Yan

West China Hospital of Sichuan University

Ying-kun Guo

West China Second University Hospital of Sichuan University

Yuan Li

West China Hospital of Sichuan University

Zhi-gang Yang ( $\square$ yangzg666@163.com)

West China Hospital of Sichuan University

\section{Research Article}

Keywords: texture analysis, cardiac amyloidosis, hypertrophic cardiomyopathy, left ventricular hypertrophy

Posted Date: June 9th, 2021

DOI: https://doi.org/10.21203/rs.3.rs-596386/v1

License: (c) (i) This work is licensed under a Creative Commons Attribution 4.0 International License. Read Full License 


\section{Abstract}

\section{Background}

To elucidate the value of texture analysis (TA) in detecting and differentiating myocardial tissue alterations on non-contrast T2-weighted CMR (cardiovascular magnetic resonance imaging) in patients with cardiac amyloidosis (CA) and hypertrophic cardiomyopathy (HCM).

\section{Methods}

In this retrospective study, $100 \mathrm{CA}(58.5 \pm 10.7$ years; 41 (41\%) females) and $217 \mathrm{HCM}(50.7 \pm 14.8$ years, 101 (46.5\%) females) patients who underwent CMR scans were included. Regions of interest for TA were delineated by two radiologists independently on non-contrast T2-weighted imaging (T2WI). Stepwise dimension reduction and texture feature selection based on reproducibility, machine learning algorithms, and correlation analyses were performed to select features. Both the CA and HCM groups were randomly divided into a training dataset and a testing dataset (7:3). After the TA model was established in the training set, the diagnostic performance of the model was validated in the testing set and further validated in a subgroup of patients with similar hypertrophy.

\section{Results}

The 7 independent texture features provided, in combination, a diagnostic accuracy of $86.0 \%$ (AUC=0.915; $95 \% \mathrm{Cl}: 0.879-0.951)$ in the training dataset and $79.2 \%$ (AUC=0.842; $95 \% \mathrm{Cl}: 0.759-0.924)$ in the testing dataset. The differential diagnostic accuracy in the similar hypertrophy subgroup was $82.2 \%$ (AUC=0.864, $95 \% \mathrm{Cl}: 0.805-0.922)$. The significance of the difference between the AUCs of the TA model and late gadolinium enhancement (LGE) was verified by Delong's test $(p=0.996)$. All seven texture features showed significant differences between CA and HCM (all $p<0.001)$.

\section{Conclusion}

Our study demonstrated that texture analysis based on non-contrast T2-weighted images could feasibly differentiate $\mathrm{CA}$ from $\mathrm{HCM}$, even in patients with similar hypertrophy. The selected final texture features could achieve a comparable diagnostic capacity to LGE.

\section{Trial registration:}

Since this study is a retrospective observational study and no intervention had been involved, trial registration is waived.

\section{Introduction}

Cardiac amyloidosis (CA) is a progressive and infiltrative cardiomyopathy that frequently leads to heart failure and cardiac death[1]. CA is characterized by left ventricular hypertrophy (LVH) and preserved left 
ventricular ejection fraction (LVEF)[2]. In certain clinical scenarios, it can still be a challenge to differentiate CA from hypertrophic cardiomyopathy $(\mathrm{HCM})$ [3]. However, it is of utmost importance to diagnose $\mathrm{CA}$ in an early stage to guide the management and improve the prognosis of these patients[4].

At present, late gadolinium enhancement (LGE) of cardiovascular magnetic resonance imaging (CMR) has remarkable diagnostic performance in diagnosing CA and HCM in clinical settings[5]. However, CA patients frequently suffer from impaired renal function, either due to amyloid deposition in the kidneys or reduced cardiac output from heart failure (HF). And the use of a gadolinium contrast agent is known to have the potential to cause renal sclerosis[6]. Thus, contrast agents are contraindicated in quite a few CA patients. In addition, both CA and HCM may not present with typical LGE patterns. A diagnosis based on LGE cannot be very definitive. Thus, using a non-contrast method for diagnosing CA and avoiding contrast-induced renal injury seems extremely important.

T2-weighted imaging (T2WI) is widely used in the clinic to identify pathological lesions in many organs, such as the brain, liver and muscles. Abnormal T2 signal intensity is correlated with edema, cellular proliferation, and vessel densities[7, 8]. In the heart, T2-weighted sequences are often used to identify myocardial edema[9, 10]. However, due to the low contrast between normal and edematous myocardium, it is often a challenge to determine the presence and extent of the lesion[11].

Texture analysis (TA) quantifies the texture of an image based on spatial distributions of pixel signal intensity and relationships of values between neighboring pixels[12]. TA has the capability to overcome the limitations of traditional subjective visual interpretation of images and recognize lesions that are imperceptible to the human eye. CMR-based TA has been reported to have great performance for several clinical applications. A previous study found that TA of T2 mapping presented high sensitivity and specificity for the diagnosis of acute infarct-like myocarditis[13].

To the best of our knowledge, this is the first study to use texture features derived from T2WI to discriminate CA from HCM. We retrospectively included $100 \mathrm{CA}$ and $217 \mathrm{HCM}$ patients, aiming to elucidate the value of TA in detecting and differentiating myocardial tissue alterations on non-contrast T2-weighted CMR images of patients with CA and HCM.

\section{Methods And Materials}

\section{Study population}

We retrospectively included 317 subjects (CA: $n=100, H C M: n=217$ ) who underwent CMR scans between January 2016 and June 2020. This study was approved by the institutional review board and in accordance with the Declaration of Helsinki. Informed consent was obtained from all participants.

CA was diagnosed on the basis of positive myocardial biopsy or extracardiac biopsy in conjunction with a mean LV wall thickness (septum and posterior wall) $\geq 12 \mathrm{~mm}$ on CMR[14]. The diagnosis of HCM was based on the presence of unexplained LV wall thickness $\geq 15 \mathrm{~mm}$ or $\geq 13 \mathrm{~mm}$ with a family history of 
HCM or apical hypertrophy in the absence of other conditions capable of producing a similar degree of hypertrophy[15]. HCM patients with previous septal ablation or myectomy were excluded.

The exclusion criteria for all subjects were valvular heart disease (greater than mild stenosis or greater than moderate regurgitation), significant coronary artery disease, and other confirmed systemic diseases. Images of poor quality were excluded. Hypertensive patients with concentric hypertrophy were also excluded.

\section{Cardiovascular magnetic resonance}

CMR imaging was performed in accordance to a standard protocol, as previously published by our group[16]. All CMR images were performed using a 3.0T scanner (MAGNETOM Skyra, Siemens Healthcare, Erlangen, Germany) with a 30-channel phased-array receiver coil. Cine images were obtained using a balanced steady-state free-precession sequence in consecutive short-axis views covering the entire LV (from the level of the mitral valve annulus to the LV apex) with the following parameters: repetition time (TR), $39.34 \mathrm{~ms}$; echo time (TE) $1.22 \mathrm{~ms}$; flip angle, 38 ; field of view (FOV), $284 \times 399 \mathrm{~mm}^{2}$; matrix size, $139 \times 208 \mathrm{~mm}^{2}$; and slice thickness, $8 \mathrm{~mm}$. T2-weighted short inversion time inversion recovery (T2-STIR) imaging was performed with the following parameters: TR, 2RR; TE, $71 \mathrm{~ms}$; flip angle, $180^{\circ}$; FOV, $243 \times 300 \mathrm{~mm}^{2}$; matrix size, 256 × $166 \mathrm{~mm}^{2}$; and slice thickness, $8 \mathrm{~mm}$. Late gadolinium enhancement (LGE) imaging was performed at an average of 10-15 min after contrast injection by using a segmented-turbo-FLASH-phase-sensitive inversion recovery (PSIR) sequence (TR/TE = 750.00/1.18 ms; flip angle $=40^{\circ}$; slice thickness $=8 \mathrm{~mm} ;$ FOV $=400 \times 270 \mathrm{~mm}^{2}$; and matrix size $=184 \times 256 \mathrm{~mm}^{2}$ ).

\section{Imaging analysis}

Conventional CMR parameters including LV end-diastolic volume (EDV), LV end-systolic volume (ESV), LVEF, LV mass, maximal LV wall thickness (MWT) and LGE extent were calculated using commercially available software (CVI ${ }^{42}$; Circle Cardiovascular Imaging, Inc., Calgary, Canada). The extent of LGE was quantified by 5 standard deviations (SDs) above the signal intensity of remote normal myocardium[17].

Texture analysis was performed on non-contrast T2-weighted CMR scans using 3D Slicer based on the Pyradiomics library[18]. The regions of interest (ROIs) were manually delineated in the basal septum of the left ventricle by a radiologist with 4 years of experience in cardiovascular imaging who was blinded to the patients' information. ROI delineation was repeated twice in a subset of 30 randomly selected patients by the same radiologist for intraobserver analysis and by another radiologist with 15 years of experience for interobserver analysis.

\section{Feature extraction and selection}

A total of 837 features were extracted from T2-weighted images during the process of image filtering and feature extraction. Stepwise feature selection and dimension reduction were performed due to the high number of texture features. First, the intraclass correlation coefficient (ICC) was calculated to assess the intra- and interobserver reproducibility of the selected features. Features with ICCs $<0.75$ were excluded, 
ICCs ranging from 0.75 to 1 considered "excellent"[19]. The Boruta algorithm[20], corrplot by carret[21] and the least absolute shrinkage and selection operator (LASSO) with 10-fold cross-validation[22] were performed in a stepwise manner for dimension reduction. Furthermore, both the CA and HCM groups were randomly divided into a training dataset and a testing dataset (7:3). To select variables that allow for the discrimination of myocardial tissue alterations in HCM and CA patients, the classification tree model[23], a commonly used machine learning algorithm, was employed to calculate discriminative performance in the training cohort and validated in the testing cohort.

After the radiomics signature was established, the diagnostic efficiency and accuracy of the model was validated in patients with similar hypertrophy matched by LV mass index as well as age, sex and maximum wall thickness (MWT).

\section{Statistics analysis}

Statistical analysis during the construction of the radiomics signature was performed in R (version 4.0.1; R Foundation for Statistical Computing, Vienna, Austria)[24] with RStudio (version 1.3.959; RStudio, Boston, Mass)[25]. The R packages used for statistical analyses are described in the supplementary materials. Other statistical analyses were conducted with SPSS (Version 19; IBM, Armonk, NY). The normality of the data distribution was determined using the Kolmogorov-Smirnov test. Continuous data are expressed as the means \pm SDs or medians with interquartile ranges. The t-test or the Mann-Whitney Utest was conducted, as appropriate. The diagnostic accuracy of the optimal radiomic parameters was evaluated by the area under the curve (AUC) from receiver operating characteristic (ROC) analyses. The diagnostic sensitivity, specificity, positive predictive value (PPV), negative predictive value (NPV) and accuracy were also calculated. ROC curves were compared using DeLong's test. $\mathrm{P}<0.05$ was considered statistically significant.

\section{Results}

\section{Clinical characteristics of the included patients}

The clinical and CMR characteristics of the 317 included patients (CA: $n=100,41 \%$ female, $58.5 \pm 10.7$ years; $\mathrm{HCM}: \mathrm{n}=217,46.5 \%$ female, $50.7 \pm 14.8$ years) are presented in Table 1 . The CA group was included based on myocardial biopsy $(n=15,15 \%)$ and extracardiac biopsy: bone morrow $(n=59,59 \%)$, fat $(n=16,16 \%)$, kidney $(n=5,5 \%)$, rectum $(n=4,4 \%)$, and tongue $(n=1,1 \%)$. In addition, $93 \%$ of the CA patients had the light-chain amyloidosis type.

The patients with CA were older and had higher NYHA functional classes than the HCM patients. The HCM group had a higher body mass index. Moreover, more hypertensive patients were found in the HCM group than in the CA group. The NT-proBNP and troponin $\mathrm{T}$ levels were markedly higher in the CA group than in the HCM group (log NT-proBNP: $8.5 \pm 1.1$ vs. $7.0 \pm 1.0$; $\log$ troponin T: $4.6 \pm 0.7$ vs. $3.0 \pm 0.9 ; p<$ 0.001 for both). 
Table 1

Demographic and clinical characteristics of the included subjects.

\begin{tabular}{|c|c|c|c|}
\hline & $C A(n=100)$ & HCM $(n=217)$ & $P$ values \\
\hline Age, years & $58.5 \pm 10.7$ & $50.7 \pm 14.8$ & $<0.001$ \\
\hline Gender, females, n(\%) & $41(41 \%)$ & $101(46.5 \%)$ & 0.318 \\
\hline BMI, $\mathrm{kg} / \mathrm{m}^{2}$ & $22.0 \pm 3.3$ & $24.1 \pm 3.5$ & $<0.001$ \\
\hline NYHA III-IV, n(\%) & $64(64 \%)$ & $23(10.6 \%)$ & $<0.001$ \\
\hline Hypertension, n(\%) & $16(16 \%)$ & $65(30 \%)$ & 0.001 \\
\hline Diabetes, n(\%) & $9(9 \%)$ & $18(8.3 \%)$ & 0.263 \\
\hline Dyslipidemia, n(\%) & $7(7 \%)$ & $12(5.5 \%)$ & 0.823 \\
\hline \multicolumn{4}{|l|}{ Sub-types of HCM } \\
\hline Asymmetrical /LOVOT & 37 & $168 / 124$ & \\
\hline Concentric & 63 & 36 & \\
\hline Mid-ventricular & / & 2 & \\
\hline Apical & / & 11 & \\
\hline$A L, n(\%)$ & $93(93 \%)$ & / & \\
\hline NT-proBNP, pg/ml & $8.5 \pm 1.1$ & $7.0 \pm 1.0$ & $<0.001$ \\
\hline Troponin T, pg/ml & $4.6 \pm 0.7$ & $3.0 \pm 0.9$ & $<0.001$ \\
\hline \multicolumn{4}{|l|}{ Medications } \\
\hline Diuretics & $68(68 \%)$ & $26(12 \%)$ & \\
\hline$\beta$-blockers & $26(26 \%)$ & $78(35.9 \%)$ & \\
\hline ACEI/ARB & $12(12 \%)$ & $31(14.2 \%)$ & \\
\hline $\mathrm{Ca}^{2+}$ blockers & $5(5 \%)$ & $33(15.2 \%)$ & \\
\hline Statin & $8(8 \%)$ & $22(10.1 \%)$ & \\
\hline \multicolumn{4}{|c|}{ Cardiovascular magnetic resonance } \\
\hline LV-ESV index, ml & $26.6 \pm 9.5$ & $23.9 \pm 9.2$ & 0.029 \\
\hline LV-EDV index, ml & $49.6 \pm 11.4$ & $59.8 \pm 13.8$ & $<0.001$ \\
\hline
\end{tabular}

CA: cardiac amyloidosis; HCM: hypertrophic cardiomyopathy; AL: light-chain amyloidosis; ESV: endsystolic volume; EDV: end-diastolic volume; LVEF: left ventricular ejection fraction; MWT: maximal wall thickness; LGE: late gadolinium enhancement. 


\begin{tabular}{|lcll|}
\hline & CA $(\mathbf{n}=100)$ & HCM $(\mathbf{n = 2 1 7 )}$ & P values \\
\hline LVEF, \% & $46.6 \pm 13.0$ & $61.0 \pm 7.9$ & $<0.001$ \\
\hline LV mass index, g/m2 & $43.3 \pm 13.3$ & $45.6 \pm 17.5$ & 0.25 \\
\hline MWT & $15.4(14.5,17.6)$ & $18.5(16.2,21.1)$ & $<0.001$ \\
\hline Presence of main LGE pattern & & & \\
\hline Patchy & $17(24.3 \%)$ & $163(75 \%)$ & \\
\hline Transmural & $25(35.7 \%)$ & $54(25 \%)$ & \\
\hline $\begin{array}{l}\text { Subendocardial } \\
\text { Diffuse }\end{array}$ & $23(32.8 \%)$ & & $<0.001$ \\
\hline $\begin{array}{l}\text { LGE extent(g) } \\
\text { CA: cardiac amyloidosis; HCM: hypertrophic cardiomyopathy; AL: light-chain amyloidosis; ESV: end- }\end{array}$ & \\
\hline $\begin{array}{l}\text { systolic volume; EDV: end-diastolic volume; LVEF: left ventricular ejection fraction; MWT: maximal } \\
\text { wall thickness; LGE: late gadolinium enhancement. }\end{array}$ & & \\
\hline
\end{tabular}

\section{Traditional CMR parameters}

The ESV index was higher in the CA group than in the HCM group (CA vs HCM: $26.6 \pm 9.5$ vs $23.9 \pm 9.2, p$ $=0.029)$, while the EDV index was higher in the HCM group ( $49.6 \pm 11.4$ vs $59.8 \pm 13.8, p<0.001)$. The LV mass index was comparable between the two groups ( $43.3 \pm 13.3$ vs $45.6 \pm 17.5, p=0.25)$, whereas the MWT was higher in the HCM group than in the CA group $(15.4[14.5,17.6]$ vs $18.5[16.2,21.1], p<0.001)$. CA patients displayed a remarkably higher LGE extent than HCM patients $(61.3[41.0,87.3]$ vs $10.6[3.5$, 30.3], $p<0.001)$.

\section{Multistep texture feature selection and dimension reduction}

The multistep texture feature selection and dimension reduction process is described in Fig. 1. In total, we extracted 837 texture features from 6 feature groups, including first-order features, gray level cooccurrence matrix (GLCM), gray level dependence matrix (GLDM), gray level run length matrix (GLRLM), gray level size zone matrix (GLSZM), and neighboring gray tone dependence matrix (NGTDM). Definitions and interpretations of the texture features are presented in the supplementary materials. Based on the ICCs for intra- and interobserver reproducibility, 614 features with ICCs $<0.75$ were first excluded. The Boruta algorithm, based on the random forest machine learning algorithm, further confirmed 51 important features. Then, a correlation matrix was calculated for these features to eliminate collinearity at the level of $\mid$ rhol $\geq 0.9$, leading to a further reduction from 51 to 20 features. These 20 features were finally fed into the LASSO algorithm, resulting in the 7 most important and independent texture features used for model fitting. 


\section{Selected texture features}

The included set of features consisted of four wavelet-transformed features (GLRLM-Long Run Emphasis (LRE) LHL; GLRLM-Short Run Emphasis (SRE) LHL; GLRLM-LRE LLH; and GLDM-Small Dependence High Gray Level Emphasis (SDHGLE) HLH) and three original features (First order-Skewness; GLDM-Large Dependence Emphasis (LDE); GLCM-Correlation). All seven texture features showed significant differences between CA and HCM (Table 2). Correlation analyses suggested that GLRLM-SRE LHL was well correlated with GLRLM-LRE LHL $(\rho=-0.85)$ and GLDM-LDE $(\rho=-0.65)$. GLDM-SDHGLE HLH had moderate correlations with GLRLM-LRE LLH $(\rho=-0.41)$, GLRLM-LRE LHL $(\rho=-0.5)$ and GLRLM-SRE LHL ( $\rho$ $=0.51$ ). The other features had weak or no correlations with each other (Fig. 2).

Table 2

Differences of Selected Texture Features Between CA and HCM

\begin{tabular}{|c|c|c|c|c|c|}
\hline $\begin{array}{l}\text { Image } \\
\text { type }\end{array}$ & $\begin{array}{l}\text { Feature } \\
\text { Class }\end{array}$ & Features names & $\mathrm{CA}$ & HCM & $\begin{array}{l}P \\
\text { value }\end{array}$ \\
\hline $\begin{array}{l}\text { wavelet- } \\
\text { LHL }\end{array}$ & GLRLM & Long Run Emphasis & $\begin{array}{l}\text { 6.639(5.796, } \\
7.310)\end{array}$ & $\begin{array}{l}7.360(6.721 \\
8.266)\end{array}$ & $\begin{array}{l}< \\
0.0001\end{array}$ \\
\hline $\begin{array}{l}\text { wavelet- } \\
\text { LHL }\end{array}$ & GLRLM & Short Run Emphasis & $\begin{array}{l}0.535(0.510 \\
0.567)\end{array}$ & $\begin{array}{l}0.504(0.478 \\
0.530)\end{array}$ & $\begin{array}{l}< \\
0.0001\end{array}$ \\
\hline $\begin{array}{l}\text { wavelet- } \\
\text { LLH }\end{array}$ & GLRLM & Long Run Emphasis & $\begin{array}{l}\text { 143.8(104.5, } \\
192.4)\end{array}$ & $\begin{array}{l}\text { 193.9(148.7, } \\
252.2)\end{array}$ & $\dot{0} 0.0001$ \\
\hline $\begin{array}{l}\text { wavelet- } \\
\text { HLH }\end{array}$ & GLDM & $\begin{array}{l}\text { Small Dependence High Gray } \\
\text { Level Emphasis }\end{array}$ & $0.143 \pm 0.019$ & $0.128 \pm 0.016$ & $\dot{0} 0001$ \\
\hline Original & $\begin{array}{l}\text { First } \\
\text { order }\end{array}$ & Skewness & $0.323 \pm 0.436$ & $0.107 \pm 0.447$ & $<0.0001$ \\
\hline Original & GLDM & Large Dependence Emphasis & $\begin{array}{l}48.3(38.8 \\
56.2)\end{array}$ & $\begin{array}{l}55.6(50.2 \\
60.0)\end{array}$ & $\dot{0} .0001$ \\
\hline Original & GLDM & Correlation & $\begin{array}{l}0.911(0.858 \\
0.951)\end{array}$ & $\begin{array}{l}0.819(0.712 \\
0.908)\end{array}$ & $<.0001$ \\
\hline
\end{tabular}

\section{Diagnostic performance of the selected texture features}

The 7 selected texture features provided, in combination, a diagnostic accuracy of $86.0 \%$ (AUC $=0.915$; $95 \% \mathrm{Cl}: 0.879-0.951)$ in the training dataset and $79.2 \%$ (AUC $=0.842 ; 95 \% \mathrm{Cl}: 0.759-0.924)$ in the testing dataset (Fig. 3). The differential diagnostic accuracy in the similar hypertrophy subgroup was $82.2 \%$ (AUC $=0.864,95 \% \mathrm{Cl}: 0.805-0.922)$ (Table 3). The significance of the difference between the AUCs of these parameters was verified by Delong's test (TA model vs. LGE, $p=0.996)$. 
Table 3

Diagnostic capacity of the selected tissue features for discriminating CA and HCM.

\begin{tabular}{|lllllll|}
\hline & AUC $(\mathbf{9 5} \% \mathrm{Cl})$ & Accuracy & Sensitivity & Specificity & NPV & PPV \\
\hline Training group & $0.915(0.879,0.951)$ & 0.860 & 75.7 & 90.7 & 89.0 & 79.1 \\
\hline Testing group & $0.842(0.759,0.924)$ & 0.792 & 60.0 & 87.9 & 82.9 & 69.2 \\
\hline Similar hypertrophy & $0.864(0.805,0.922)$ & 0.822 & 83.5 & 81.0 & 83.1 & 81.5 \\
\hline AUC: the area under the curve, PPV: positive predictive value, NPV: negative predictive value & \\
\hline
\end{tabular}

\section{Intraobserver and interobserver reproducibility}

The ICCs of the 837 radiomics features ranged from 0 to 0.983 . Among them, 614 features had an ICC < 0.75 and were excluded from further analyses. The reproducibility of the final selected 7 features was considered excellent (ICCs ranging from 0.757 to 0.949 ) (Table 4).

Table 4

Intraclass correlation coefficients for the intra- and interobserver reproducibility of the selected texture features

\begin{tabular}{|lccccccc|}
\hline & $\begin{array}{l}\text { GLRLM- } \\
\text { LRE LHL }\end{array}$ & $\begin{array}{l}\text { GLRLM- } \\
\text { SRE LHL }\end{array}$ & $\begin{array}{l}\text { GLRLM- } \\
\text { LRE LLH }\end{array}$ & $\begin{array}{l}\text { GLDM- } \\
\text { SDHGLE } \\
\text { LHL }\end{array}$ & $\begin{array}{l}\text { First order- } \\
\text { Skewness }\end{array}$ & $\begin{array}{l}\text { GLDM- } \\
\text { LDE }\end{array}$ & $\begin{array}{l}\text { GLDM- } \\
\text { Correlation }\end{array}$ \\
\hline $\begin{array}{l}\text { Intra- } \\
\text { observer }\end{array}$ & 0.949 & 0.893 & 0.855 & 0.847 & 0.880 & 0.794 & 0.773 \\
\hline $\begin{array}{l}\text { Inter- } \\
\text { observer }\end{array}$ & 0.918 & 0.883 & 0.834 & 0.761 & 0.858 & 0.758 & 0.757 \\
\hline \multicolumn{2}{l|l}{ Abbreviations as in Table 2. } & & & & & & \\
\hline
\end{tabular}

\section{Discussion}

Texture analysis is a postprocessing method to identify subtle tissue alterations and can be applied to standard and routinely acquired clinical CMR sequences. Our study demonstrated that TA was feasible and reproducible for detecting myocardial tissue alterations on non-contrast T2-weighted images. The radiomics model constructed with texture features derived from this sequence had a great performance in differentiating between CA and HCM patients and was comparable to LGE. Thus, TA of T2WI can help eliminate the need for contrast agent administration in these patients. Radiomics utilizes TA to comprehensively and elaboratively analyze the spatial distributions of pixel gray levels in images, which further derives substantial quantitative texture features characterizing the underlying tissue texture[26]. These texture features, in combination with robust mathematical models, could represent reliable diagnostic tools[27]. 
Our study indicated that the optimal combination of texture features had an accuracy of $86 \%$ for differentiating between $\mathrm{CA}$ and HCM. HCM often shows extremely and heterogeneously increased wall thickness. We validated the diagnostic capacity of the TA model in patients with similar hypertrophy matched by LV mass index, age, sex, and MWT. This subgroup analysis showed that the TA model still had a great discriminative capacity with high sensitivity and specificity for CA and HCM. Delong's tests of the AUCs showed that the diagnostic capacity of the TA model was comparable to that of LGE.

LGE is the most popular and useful CMR sequence for the diagnosis and differentiation of CA and HCM in the clinical setting. However, the required administration of a gadolinium agent has limited some patients from undergoing this examination. There are many researchers seeking to develop novel and gadolinium-free techniques for the characterization of myocardial diseases. For example, Neisius et al. utilized texture features of the myocardium through native T1 mapping to discriminate between HCM and hypertensive heart disease[28]. Baessler et al. indicated that TA of non-contrast cine images allowed for the diagnosis of subacute and chronic ischemic scars with high accuracy[29]. Our study made an effort to apply TA to non-contrast T2 images and achieved a comparable result to LGE in discriminating CA from HCM.

$\mathrm{T} 2 \mathrm{WI}$ is a routinely applied and widely accessible sequence for CMR in most institutions. A high signal intensity on T2WI is indicated to be correlated with myocardial edema and considered helpful for the diagnosis of myocarditis and acute myocardial infarction[30]. Hen et al. had previously reported that a high T2 signal was an independent predictor of life-threatening arrhythmic events in HCM patients[31]. Kotecha et al. demonstrated that myocardial edema was present in CA by histology and CMR T2 mapping. T2 signal was an independent predictor of death in light-chain amyloidosis (AL), suggesting that in addition to amyloidosis infiltration, myocardial edema possibly caused by amyloidosis fibril toxicity would be an additional mechanism contributing to mortality[32]. Our study demonstrated that texture features derived from T2WI could reflect tissue alterations of the myocardium in CA and HCM.

The most important texture feature for discriminating between CA and HCM on T2WI in our study was GLRLM-SRE LHL. A greater value of GLRLM-SRE represents finer textural textures, while a greater value of GLRLM-LRE indicates coarser structural textures. Both features are derived from the run-length feature matrix, which describes the number of gray level runs of various lengths. A gray level run is defined as a group of pixels that have the same gray-level value in a given direction. The length is the number of pixels within the run. The run-length matrix elements describe the number of runs of a specific gray level value, and a particular run length can be observed in the ROI[33]. Thus, the two features GLRLM-SRE and GLRLM-LRE can serve as a measure of tissue homogeneity. In our study, the CA group showed lower GLRLM-LRE and higher GLRLM-SRE values than the HCM group, suggesting that CA has a finer textural texture on T2WI. The possible reason for this might be correlated with the more pronounced myocardial edema present in $\mathrm{CA}$ [32]. The coarse texture of $\mathrm{HCM}$ might reflect tissue inhomogeneity, such as myocardial disarray and fibrosis. Thus, TA seems to be a useful quantitative tool for the identification of tissue alterations. 


\section{Limitations}

This study still had several limitations. First, this was a single-center study. Second, since this was a retrospective study, texture analysis of novel sequences, such as T1 mapping and T2 mapping, could not be performed at this time. However, we will conduct prospective studies with these novel imaging techniques in the future. In addition, the sub-analysis of patients with similar hypertrophy was limited by the small sample size. Further well-designed prospective studies are necessary to determine the utility of these TA parameters for a more general application.

\section{Conclusion}

In conclusion, our study demonstrated that texture analysis based on non-contrast T2-weighted images can feasibly differentiate CA from HCM, even in patients with similar hypertrophy. The selected final texture features could achieve a comparable diagnostic capacity to LGE. TA could help eliminate the use of contrast agents and additional sequences to discriminate between these two groups of patients in clinical settings.

\section{Abbreviations}

CA: Cardiac amyloidosis; HCM: Hypertrophic cardiomyopathy; CMR: Cardiovascular magnetic resonance imaging; T2WI: T2-weighted imaging; TA: Texture analysis; LGE: Late gadolinium enhancement; EDV: LV end-diastolic volume; ESV: LV end-systolic volume; LVEF: Left ventricular ejection fraction; MWT: Maximal LV wall thickness; LASSO: The least absolute shrinkage and selection operator; GLCM: Gray level cooccurrence matrix; GLDM: Gray level dependence matrix; GLRLM: Gray level run length matrix; GLSZM: Gray level size zone matrix; NGTDM: Neighboring gray tone dependence matrix; LRE: Long Run Emphasis; SRE: Short Run Emphasis; SDHGLE: Small Dependence High Gray Level Emphasis; LDE: Large Dependence Emphasis;

\section{Declarations}

\section{Ethics approval and consent to participate}

This study was approved by the Institutional Ethics Committee of West China Hospital, Sichuan University (Chengdu, Sichuan, China) and conducted in accordance with the Declaration of Helsinki. Informed consent was obtained from all participants.

\section{Consent for publication}

Not applicable.

\section{Availability of data and materials}

The datasets analyzed in the current study are available from the corresponding author on reasonable request. 


\section{Funding}

This work was supported by $1 \cdot 3.5$ project for disciplines of excellence, West China Hospital, Sichuan University (ZYGD18013).

\section{Authors' contributions}

S.H., Z.G.Y and Y.L were the major contributors to the study design, manuscript drafting and revisions. K.S. and S.H. recruited the subjects and collected clinical data of the included subjects. Y.Z and W.F.Y. analysed the traditional CMR parameters and interpret the results. S.H and Y.L. delineated regions of interest on the T2 images and extracted the texture features. K.S and Y.Z performed the statistical analyses. W.F.Y prepared the figures. Z.G.Y. and Y.K.G. jointly supervised the whole work and revised the manuscript. All authors critically revised the manuscript for important intellectual content and approved the final manuscript.

\section{Acknowledgements}

NA

\section{References}

1. Falk Rodney H. Diagnosis and management of the cardiac amyloidoses. Circulation 2005;112:2047.

2. Esther G-L, Maria GD, Gonzalo GM, Javier HDMF, Marta CM, Carolina R, et al. Wild-type transthyretin amyloidosis as a cause of heart failure with preserved ejection fraction. Eur Heart J 2585-94.

3. Thibaud, Damy, Bruno, Costes, Albert A, Hagège, et al. Prevalence and clinical phenotype of hereditary transthyretin amyloid cardiomyopathy in patients with increased left ventricular wall thickness. Eur Heart J 2016.

4. Falk RH, Alexander KM, Liao R, Dorbala S. AL (Light-Chain) Cardiac Amyloidosis: a review of diagnosis and therapy. J Am Coll Cardiol 2016;68:1323-41. doi: 10.1016/j.jacc.2016.06.053.

5. Kwong RY, Falk RH. Cardiovascular Magnetic Resonance in Cardiac Amyloidosis. Circulation 2005;111:122-4. doi: 10.1161/01.CIR.0000153623.02240.20.

6. Canavese C, Mereu MC, Aime S, Lazzarich E, Fenoglio R, Quaglia M, et al. Gadolinium-associated nephrogenic systemic fibrosis: the need for nephrologists' awareness. J Nephrol 2008;21:324.

7. Togao O, Yoshiura T, Mihara F, Noguchi T, Hiwatashi A, Yamashita K, et al. Cortical thickness difference across the central sulcus visualized in the presence of vasogenic edema. Eur $\mathrm{J}$ Radiol 2008;66:274-81.

8. Inoue E, Kuroda C, Narumi Y, Fujita M, Kadota T, Kuriyama K, et al. Magnetic resonance imaginghistologic correlation of small hepatocellular carcinomas adenomatous hyperplasias. Invest Radiol 1993;28:691-7. doi: 10.1097/00004424-199308000-00007. 
9. Friedrich MG, Sechtem U, Schulz-Menger J, Holmvang G, Alakija P, Cooper LT, et al. Cardiovascular magnetic resonance in myocarditis: A JACC White Paper. J Am Coll Cardiol 2009;53:1475-87. doi: 10.1016/j.jacc.2009.02.007.

10. Abdelaty H, Zagrosek A, Schulzmenger J, Taylor AJ, Messroghli D, Kumar A, et al. Delayed enhancement and T2-weighted cardiovascular magnetic resonance imaging differentiate acute from chronic myocardial infarction. Circulation 2004;13:25-25.

11. Amano Y, Tachi M, Tani H, Mizuno K, Kobayashi Y, Kumita S. T2-weighted cardiac magnetic resonance imaging of edema in myocardial diseases. ScientificWorldJournal 2012;2012:194069. doi: 10.1100/2012/194069.

12. MacKay JW, Murray PJ, Kasmai B, Johnson G, Donell ST, Toms AP. MRI texture analysis of subchondral bone at the tibial plateau. Eur Radiol 2016;26:3034-45. doi: 10.1007/s00330-015-41420 .

13. Baessler B, Luecke C, Lurz J, Klingel K, von Roeder M, de Waha S, et al. Cardiac MRI Texture Analysis of T1 and T2 Maps in Patients with Infarctlike Acute Myocarditis. Radiology 2018;289:357-65. doi: 10.1148/radiol.2018180411.

14. Gertz MA, Comenzo R, Falk RH, Fermand JP, Hazenberg BP, Hawkins PN, et al. Definition of organ involvement and treatment response in immunoglobulin light chain amyloidosis (AL): A consensus opinion from the 10th International Symposium on Amyloid and Amyloidosis. Am J Hematol 2005;79:319-28. doi: 10.1002/ajh.20381.

15. Perry M. Elliott, Michael A. Borger, Martin Borggrefe, Franco Cecchi, Philippe Charron, Albert Alain Hagege, et al. 2014 ESC Guidelines on diagnosis and management of hypertrophic cardiomyopathy: The Task Force for the Diagnosis and Management of Hypertrophic Cardiomyopathy of the European Society of Cardiology (ESC). Eur Heart J 2014;35:2733-79. doi: 10.1093/eurheartj/ehu284.

16. Huang S, Xu H, Diao K, Shi K, He Y, He S, et al. Left ventricular global function index by magnetic resonance imaging - a novel marker for differentiating cardiac amyloidosis from hypertrophic cardiomyopathy. Sci Rep 2020;10:4707. doi: 10.1038/s41598-020-61608-9.

17. Schulz-Menger J, Bluemke DA, Bremerich J, Flamm SD, Fogel MA, Friedrich MG, et al. Standardized image interpretation and post processing in cardiovascular magnetic resonance: Society for Cardiovascular Magnetic Resonance (SCMR) Board of Trustees Task Force on Standardized Post Processing. J Cardiovasc Magn Reson 2013;15:35-53. doi: 10.1186/1532-429X-15-35.

18. van Griethuysen JJM, Fedorov A, Parmar C, Hosny A, Aucoin N, Narayan V, et al. Computational Radiomics System to Decode the Radiographic Phenotype. Cancer Res 2017;77:e104-7. doi: 10.1158/0008-5472.CAN-17-0339.

19. Khan JN, Singh A, Nazir SA, Kanagala P, Gershlick AH, Mccann GP. Comparison of cardiovascular magnetic resonance feature tracking and tagging for the assessment of left ventricular systolic strain in acute myocardial infarction. Eur J Radiol 2015;84:840-8. 
20. Kursa MB, Rudnicki WR. A deceiving charm of feature selection: The microarray case study. In: ManMachine Interactions 2. Springer; 2011: 145-52.

21. Kuhn M. Caret: classification and regression training. ascl 2015:ascl-1505.

22. Tibshirani R. Regression shrinkage and selection via the lasso: a retrospective. J R Statal Soc Ser B Statal Methodol 2011;73.

23. Therneau TM, Atkinson EJ, Foundation M. An Introduction to Recursive Partitioning Using the RPART Routines. 67.

24. Team RC, others. R: A language and environment for statistical computing. Vienna, Austria; 2013.

25. Team Rs, others. RStudio: integrated development for R. RStudio Inc Boston MA URL Httpwww Rstudio Com 2015;42:14.

26. Cendes GCastellanolBonilhalMLif. Texture analysis of medical images. Clin Radiol 2004.

27. Wang S, Summers RM. Machine learning and radiology. Med Image Anal 2012;16:933-51.

28. Neisius U, El-Rewaidy H, Nakamori S, Rodriguez J, Manning WJ, Nezafat R. Radiomic Analysis of Myocardial Native T1 Imaging Discriminates Between Hypertensive Heart Disease and Hypertrophic Cardiomyopathy. JACC Cardiovasc Imaging 2019;12:1946-54. doi: 10.1016/j.jcmg.2018.11.024.

29. Baessler B, Mannil M, Oebel S, Maintz D, Alkadhi H, Manka R. Subacute and Chronic Left Ventricular Myocardial Scar: Accuracy of Texture Analysis on Nonenhanced Cine MR Images. Radiology 2018;286:103-12. doi: 10.1148/radiol.2017170213.

30. Francone M, Chimenti C, Galea N, Scopelliti F, Verardo R, Galea R, et al. CMR Sensitivity Varies With Clinical Presentation and Extent of Cell Necrosis in Biopsy-Proven Acute Myocarditis. JACC Cardiovasc Imaging 2014;7:254-63. doi: 10.1016/j.jcmg.2013.10.011.

31. Hen Y, Takara A, Iguchi N, Utanohara Y, Teraoka K, Takada K, et al. High Signal Intensity on T2Weighted Cardiovascular Magnetic Resonance Imaging Predicts Life-Threatening Arrhythmic Events in Hypertrophic Cardiomyopathy Patients. Circ J 2018;82:1062-9. doi: 10.1253/circj.CJ-17-1235.

32. Kotecha T, Martinez-Naharro A, Treibel TA, Francis R, Nordin S, Abdel-Gadir A, et al. Myocardial Edema and Prognosis in Amyloidosis. J Am Coll Cardiol 2018;71:2919-31. doi: 10.1016/j.jacc.2018.03.536.

33. Larroza A, Bodí V, Moratal D. Texture Analysis in Magnetic Resonance Imaging: Review and Considerations for Future Applications. In: Constantinides C, ed. Assessment of Cellular and Organ Function and Dysfunction using Direct and Derived MRI Methodologies. InTech; 2016.

\section{Figures}




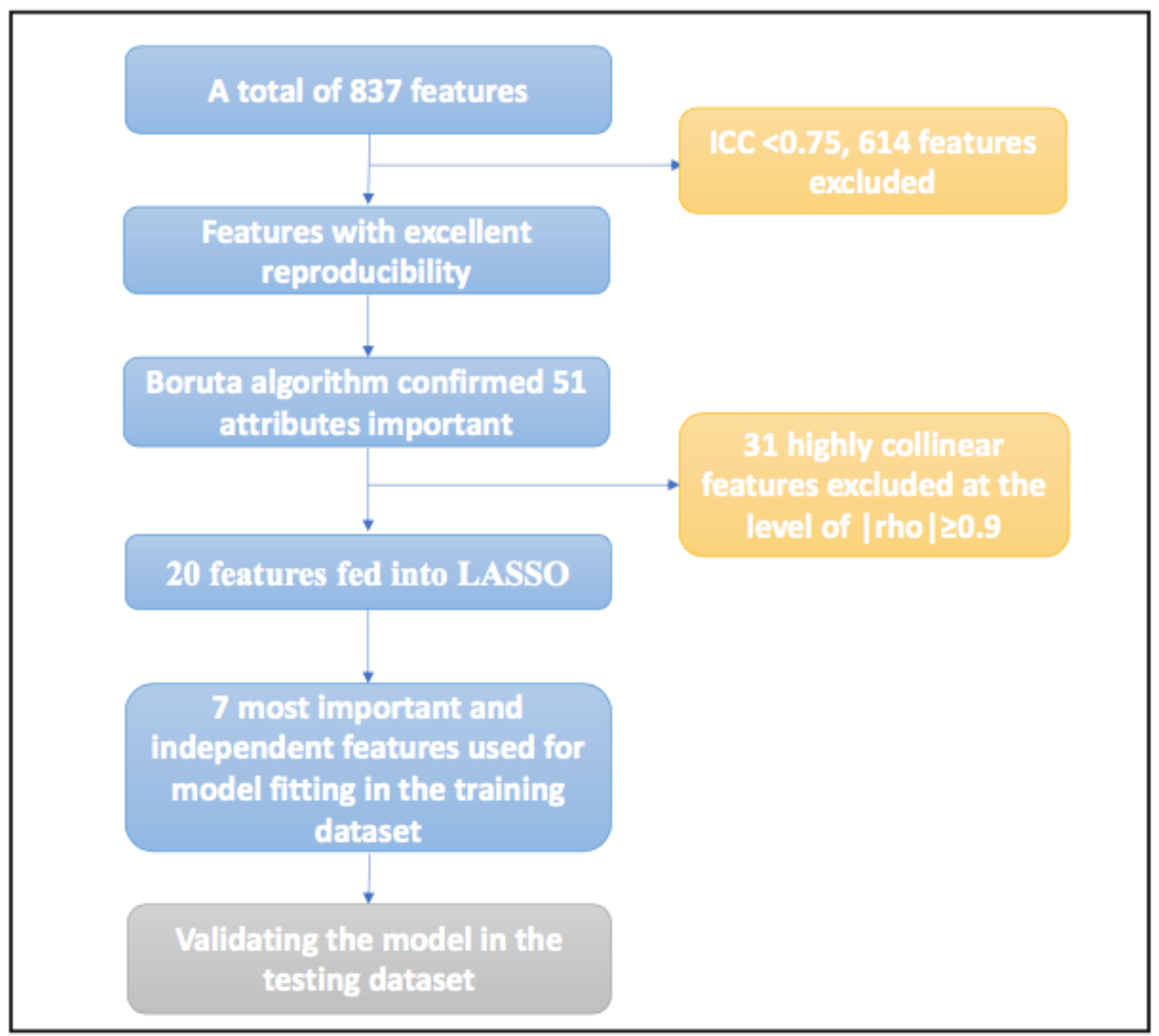

\section{Figure 1}

Feature selection and dimension reduction process. ICC: intraclass correlation coefficient, LASSO: the least absolute shrinkage and selection operator. 


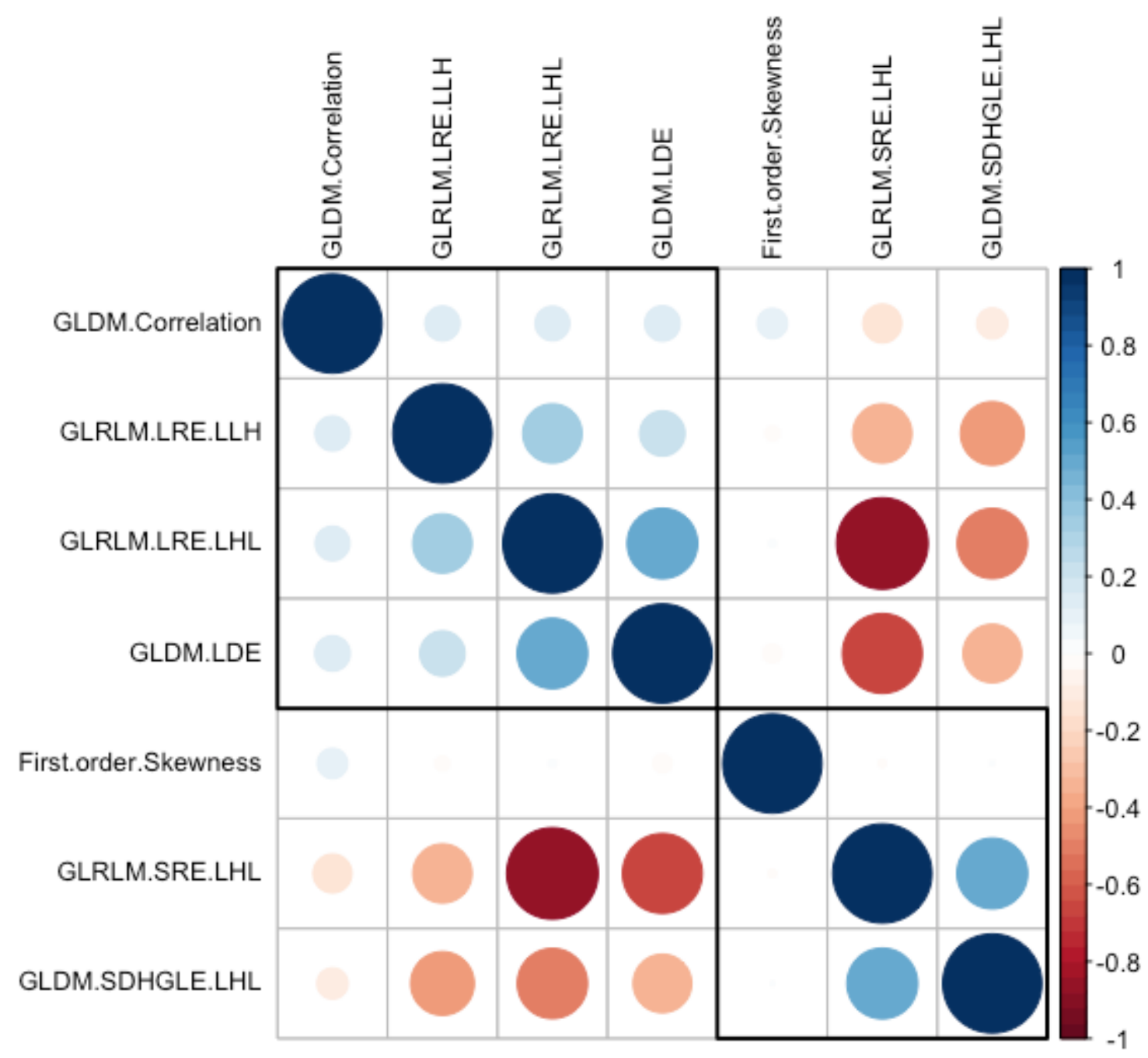

Figure 2

Correlogram of the relationship among the selected texture features. Smaller and/or lighter circles represent lower correlation. On the contrary, larger and/or darker circles indicate higher correlation. GLRLM: gray level run length matrix, GLDM: gray level dependence matrix, GLCM: gray level cooccurrence matrix; LRE: Long Run Emphasis; SRE: Short Run Emphasis; SDHGLE: Small Dependence High Gray Level Emphasis; LDE: Large Dependence Emphasis; H: high wavelet filter; L: low wavelet filter 


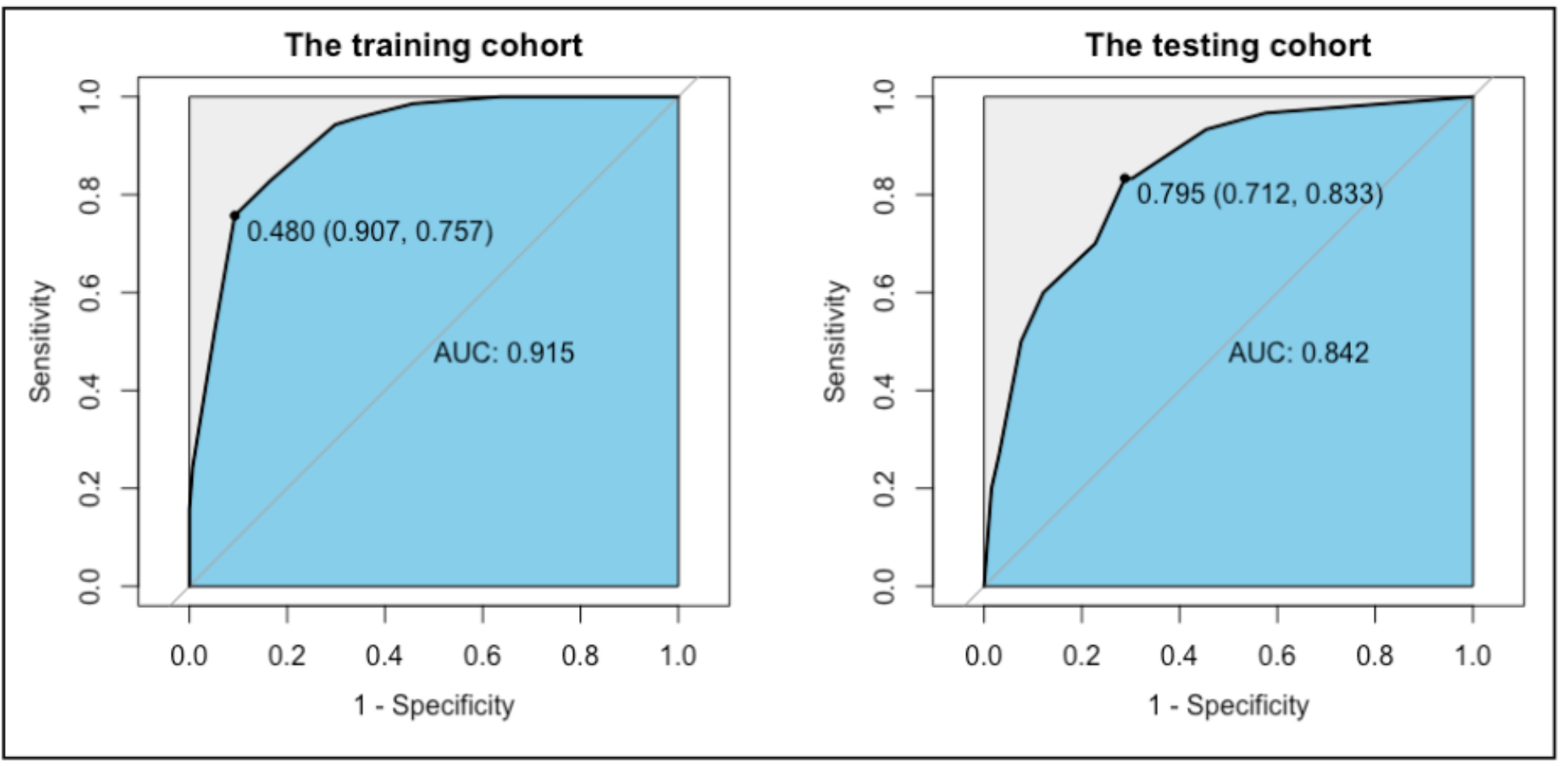

\section{Figure 3}

Receiver operating characteristic curves (ROC) for the radiomics model in differentiating cardiac amyloidosis (CA) from hypertrophic cardiomyopathy ( $\mathrm{HCM}$ ) in the training and testing cohorts. AUC: area under the ROC curve.

\section{Supplementary Files}

This is a list of supplementary files associated with this preprint. Click to download.

- Supplementarymaterials.docx 